\title{
Analysis of Light Scattering from Surface Roughness in Hollow- Core Photonic Bandgap Fibers
}

\author{
Eric Numkam Fokoua, Francesco Poletti and David J. Richardson \\ Optoelectronics Research Centre, University of Southampton, SO17 1BJ, Southampton, UK \\ ernf1g10@orc.soton.ac.uk
}

\begin{abstract}
We present a theoretical method that combines statistical information from surface roughness, mode field distribution and fibre geometry to accurately describe roughness scattering in hollow-core photonic bandgap fibres. The method predicts angular distributions of scattered power and attenuation values that agree well with experimental data.
\end{abstract}

\section{Introduction}

Hollow-core photonic bandgap fibers (HCPBGFs) confine and guide light in an air core by means of an out-of-plane photonic bandgap. As a consequence, the conventional loss mechanisms of Rayleigh scattering and phonon absorption have a lower contribution to the overall attenuation. This raises the possibility of producing HC-PBGFs with very low loss values, which together with a three orders of magnitude decrease in effective nonlinearity and a much reduced latency make HC-PBGFs an interesting candidate for data transmission.

However, the lowest loss value reported to date for HC-PBGFs stands at $1.7 \mathrm{~dB} / \mathrm{km}^{1}$, more than six times higher than in typical single mode fibers. Indeed, the many air-glass interfaces present within HC-PBGFs are intrinsically rough due to frozen-in surface capillary waves (SCWs), which are of thermodynamic origin. Scattering from these rough surfaces has been found to dominate attenuation in HC-PBGFs. Unlike Rayleigh scattering which is solely determined by the material choice, surface scattering also crucially depends on the specific fiber design. Enlarging the air core or moving the photonic bandgap to longer wavelengths for example are known ways to reduce the roughness scattering loss.

To enhance our physical understanding and advance the design of ultra-low loss HC-PBGFs, an accurate mathematical description of the scattering process is needed. The standard theoretical treatment of scattering from waveguide imperfections considers the scattering as coupling between the guided mode and all the radiation modes of the structure ${ }^{2}$. Roberts et al. have applied such an approach to solid-core photonic crystal fibers ${ }^{3}$, but the method involves complicated Green tensor math which in practice limits its applicability to structures with circular air holes. Extending previous work ${ }^{4}$ we have recently formulated a simpler method in which the far-field distribution of the scattered light is calculated using dipole radiation theory ${ }^{5}$. Our method was shown to accurately predict the scattering from both a single tube waveguide and a solid-core PCF.

Here we extend our method to the study of HC-PBGFs with realistic, non-circular air holes and show that it can predict the angular distribution of scattered light and the overall attenuation of the fiber with good accuracy. We also show that the predicted wavelength dependence agrees well with reported experimental data. Finally, we highlight the strong dependence of the loss on the roughness power spectral density, by showing that surfaces with the same value of root mean square roughness but different power density spectra can yield very different attenuation values.

\section{Derivation}

As shown in Fig.1(a) the optical field of airguided modes in HC-PBGFs have-some small overlap with the surrounding glass surfaces which generates scattered radiation and hence loss. Using coherent dipole radiation theory, it can be shown that the field scattered from a given mode out of a fiber section of length $2 L$ at point $P(\vartheta, \phi)$ on a distant sphere (see Fig.1(b)) is given by :

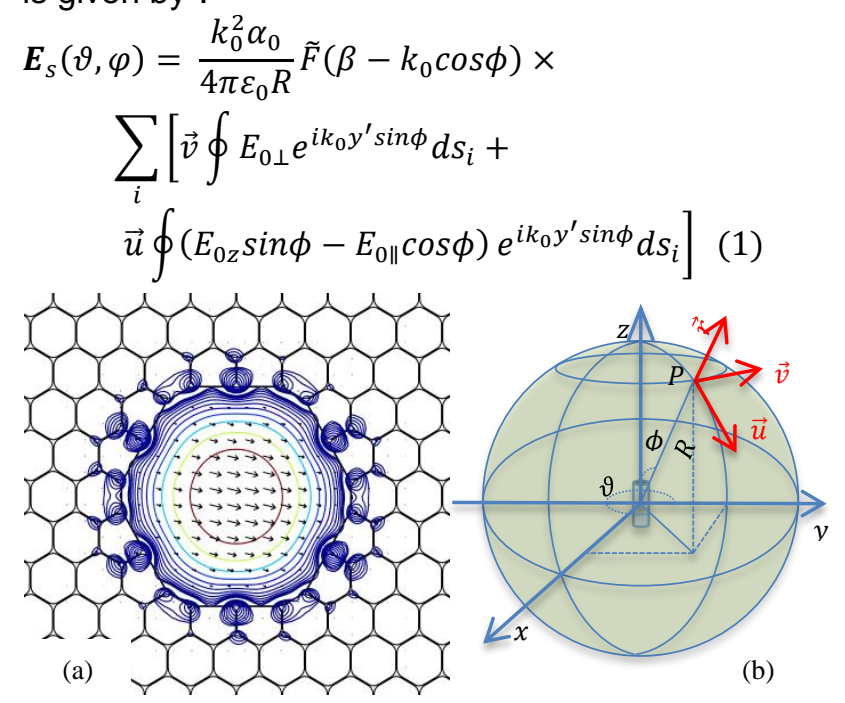

Fig. 1: (a) Mode field distribution in HC-PBGF (b) Illustration of observing point $\mathrm{P}$ on distant sphere 
This expression is the product of three separate components, which depend on (i) the wavelength $\left(k_{0}\right)$ and refractive index contrast $\left(\alpha_{0}\right)$, (ii) the Fourier transform $\tilde{F}$ of the roughness perturbation and (iii) the geometry of the waveguide and the strength of the incident electric field at the air-glass interfaces $\left(\boldsymbol{E}_{0}\right)$. The sum is over all the scattering surfaces in the cross-section, corresponding to the individual air holes in an HC-PBGF, as shown in Fig.1(a). Equation (1) also reveals that scattering at a particular angle $\phi$ is determined by the amplitude of the roughness power spectral density (PSD) at the spatial frequency $\beta-$ $k_{0} \cos \phi$, a result that conforms to the analysis of coupled-mode theory.

From the scattered field of equation (1), we can obtain the average angular distribution of scattered power typically measured in angularly resolved scattering (ARS) experiments ${ }^{1,3}$ :

$$
P(\phi)=\frac{1}{2 \pi} \int_{0}^{2 \pi} \frac{1}{2} c n \varepsilon_{0}\left|E_{s}(\vartheta, \phi)\right|^{2} R^{2} \sin \phi d \vartheta
$$

To quantify the roughness of a surface, the height profile is typically measured by either AFM or optical techniques, and statistical parameters are obtained by processing such data. Ultimately, the information that is required in Equation (2) is the roughness power spectral density (PSD), the Fourier transform of its autocorrelation function:

$$
\begin{aligned}
S(\kappa) & =\left\langle\lim _{L \rightarrow \infty} \frac{1}{2 L}\left|\int_{-L}^{L} f(z) e^{-i \kappa z} d z\right|^{2}\right\rangle \\
& =\frac{1}{2 L}|\tilde{F}(\kappa)|^{2}
\end{aligned}
$$

The exponential scattering loss coefficient is obtained by integrating (2) over all angles:

$$
\alpha_{S C}=\frac{1}{2 L P_{0}} 2 \pi \int_{0}^{\pi} P(\phi) d \phi,
$$

where $P_{0}$ is the incident power. Note that in deriving Equation (1), we have assumed that the scattered light propagates in air, which is a reasonable approximation within the microstructure area. In all HC-PBGFs however, the microstructure is surrounded by a silica outer cladding, and in ARS experiments, an index-matched fluid is often used between the fiber and a detector. In this case, the scattered light is refracted at the interface between the microstructure cladding and the silica outer jacket. The measured scattering angle $\phi_{m}$ is therefore given by Snell's law:

$$
k_{c l} \cos \phi_{m}=k_{0} \cos \phi
$$

\section{Roughness scattering in HC-PBGFs}

To model surface scattering in HC-PBGFs, we first obtain the electric field intensity at all the air-glass interfaces using a fully vectorial finite element method, and then incorporate a realistic model of the surface roughness inside the fiber to compute the far-field distribution of scattered light from equation (1). As already mentioned, frozen-in SCWs have been identified as the origin of the intrinsic roughness of the air-glass interfaces in HC-PBGFs. Their PSD is given by the well-known expression:

$$
S(\kappa)=\frac{k_{B} T_{g}}{4 \pi \gamma \kappa}
$$

where $k_{B}$ is Boltzmann's constant, $T_{g}(\sim 1500 K)$ the glass transition temperature and $\gamma\left(\sim 1 \mathrm{~J} . \mathrm{m}^{-2}\right)$ the surface tension. This expression implies a logarithmic roughness autocorrelation which requires a low spatial frequency cut-off to make physical sense. Here for simplicity we will assume that the roughness frequencies below a given cut-off frequency $\kappa_{c}\left(0.1 \mu \mathrm{m}^{-1}\right)$ have all the same PSD value. Using this assumption, we plot in Fig. 2(a) the calculated roughness scattering loss across the whole photonic bandgap for the fundamental mode of the low-loss HC-PBGF we recently fabricated ${ }^{6}$. As can be seen, our model suggests that the total loss is dominated by the roughness scattering contribution near the center of the bandgap, while the bandgap edges are determined by confinement loss. The calculated scattering loss is within a factor of 2 of the measured value, which is an excellent agreement considering the assumptions made in the derivation of the model. For more insight, we show in Fig. 2(b) the angular distribution of scattered power $P(\phi) / P_{0}$ for a number of guided modes within the fiber. The curve for the
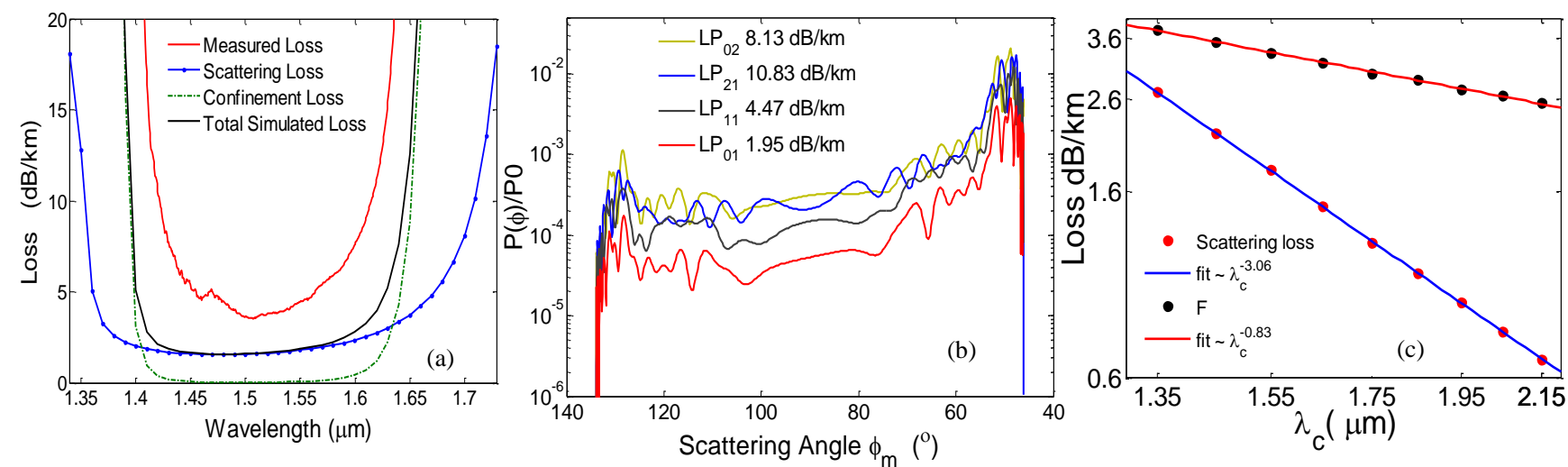

Fig. 2: (a) Comparison of calculated loss contributions to measurement (b) Angular distribution of scattered power for a few guided modes and (c) Predicted wavelength dependence of scattering loss. 
fundamental mode shows good qualitative agreement with the reported data from Roberts et al', referring to a not too dissimilar fiber design. Our model goes one step further in allowing us to fully evaluate the differential scattering loss between the guided modes of the fiber, which as can be seen can be nonnegligible for these fibers.

A crucial factor determining the ultimate loss performance of HC-PBGFs is the wavelength dependence of their scattering loss. It has been demonstrated experimentally ${ }^{1}$ that when the fiber is scaled so that the photonic bandgap is centered at longer wavelengths the loss decreases as $\lambda_{c}^{-3}$. However, a theoretical understanding of this dependence has not hitherto been provided. Our model predicts a scattering loss that indeed follows a $\lambda_{c}^{-a}$ trend, with $a=3.06$ in the case of our low-loss HCPBGF, as shown in Fig. 2(c), but which in general ranges between 2.5 and 3.5 for other designs. This suggests that for each set of design parameters the amount of field at the interfaces changes differently with wavelength. On the same figure, we also show how the normalized interface field intensity ( $F$-parameter) which is routinely used to compare fiber designs ${ }^{1}$ cannot explain the observed wavelength dependence of the loss. We believe this stems from the fact that as the operational wavelength is increased the scattering becomes influenced by lower spatial frequencies of the roughness. Therefore only models fully accounting for the exact roughness PSD - and not just using its $r m s$ value - can predict the wavelength dependence of the loss.

\section{Loss and Roughness PSD}

Besides SCWs, which as already experimentally measured with AFM measurements dominate the roughness spectrum in $\mathrm{HC}^{-P B G F s}{ }^{1}$ between spatial frequencies 0.2 and $30 \mu \mathrm{m}^{-1}$, other sources of roughness may be present at frequencies below those practically measurable. These additional roughness components, corresponding to long range waviness and possibly originating from drawing parameter fluctuations during fiber fabrication, may ultimately have an effect on the overall loss. To investigate this, here we also study the loss that would be produced in the previous HC-PBGF by roughness components with an exponential auto-correlation, $R(u)=\sigma^{2} \exp \left(-|u| / L_{c}\right)$,

where $L_{c}$ is the correlation length and $\sigma$ the roughness rms. The PSD arising from this autocorrelation function is a Lorentzian, $S(\kappa)=2 \sigma^{2} L_{c} /\left(1+L_{c}^{2} \kappa^{2}\right)$. Fig. 3 shows the predicted loss contribution from our model

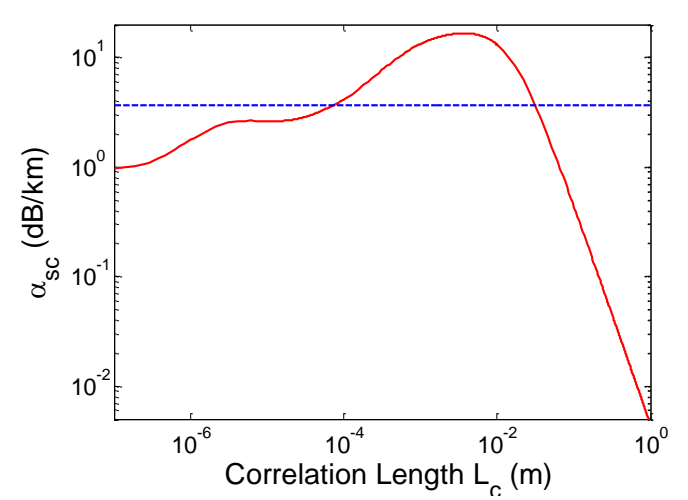

Fig. 3: Predicted scattering loss from exponential roughness. Dashed line is measured loss value.

arising from such a surface roughness as a function of $L_{c}$ and for $\lambda=1.55 \mu \mathrm{m}$, assuming $\sigma \sim 0.1 \mathrm{~nm}$. The loss increases with $L_{c}$, peaks at $L c \sim 4 \mathrm{~mm}$ and then decreases quickly, as typically observed with exponentially rough surfaces $^{2}$. Note that in a broad range of correlation lengths $(70 \mu \mathrm{m}-3.7 \mathrm{~cm})$ the scattering loss from such a roughness component alone would be higher than the measured value. If we suppose that in our HCPBGF such a long-range exponential roughness component with $L_{c}$ of the order of a few centimeters is present in addition to SCWs, we would obtain a scattering loss very close to the measured $3.5 \mathrm{~dB} / \mathrm{km}$. However the contribution of this additional roughness component would negligible in the range of AFM-measurable spatial frequencies. This shows that incorporation of accurate roughness information in the low spatial frequency region in our model could yield even more accurate loss predictions.

\section{Conclusions}

We have derived a theoretical method based on dipole radiation to describe light scattering from surface roughness in HC-PBGFs. Our method not only returns an accurate value of loss, but also accurately predicts the angular distribution of scattered power. Using our model, the predicted loss from frozen-in SCWs alone is within a factor of 2 of the measured value. An even closer prediction of the loss can be obtained if other realistic long-range perturbations are incorporated into the model.

Acknowledgements: This work was supported by the EU program FP7-ICT MODE-GAP.

\section{References}

[1] P.J Roberts et al, Opt.Exp.13, 236 (2005)

[2] D. Marcuse, Theory of Dielectric optical waveguides, Academic Press 1991

[3] P.J Roberts et al, Opt. Exp. 13, 7779 (2005)

[4] E.G. Rawson, Appl. Opt. 13, 2370 (1974)

[5]Numkam et al, Proc. OFC'12, JW2A.18(2012)

[6] Wheeler et al, Proc. OFC'12 PDP5A.2 (2012) 